\title{
AN INJECTOR FOR THE PROPOSED BERKELEY ULTRAFAST X-RAY LIGHT SOURCE*
}

\author{
S. Lidia ${ }^{\#}$, J. Corlett, J. Pusina, J. Staples, A. Zholents, Lawrence Berkeley National Laboratory, \\ Berkeley, CA 94720, USA
}

\section{Abstract}

Berkeley Lab has proposed to build a recirculating linac based $\mathrm{x}$-ray source for ultra-fast dynamic studies [1]. This machine requires a flat electron beam with a small vertical emittance and large $\mathrm{x} / \mathrm{y}$ emittance ratio to allow for compression of spontaneous undulator emission of soft and hard x-ray pulses, and a low-emittance, round electron beam for coherent emission of soft $\mathrm{x}$-rays via the FEL process based on cascaded harmonic generation [2]. We propose an injector system consisting of two high gradient high repetition rate photocathode guns [3] (one for each application), a $\sim 120 \mathrm{MeV}$ superconducting linear accelerator, a $3^{\text {rd }}$ harmonic cavity for linearization of the longitudinal phase space, and a bunch compressor. We present details of the design and the results of particle tracking studies using several computer codes.

\section{INTRODUCTION}

The proposed Linac-based Ultra-fast X-ray source ('LUX') facility [1] requires a high-brightness, high duty factor, and highly reliable electron beam injector to produce both hard $\mathrm{x}$-rays by spontaneous emission in undulators and wigglers and soft $\mathrm{x}$-rays in cascaded harmonic generation free-electron lasers. Two rf photoinjector guns produce electron beams with very different emittance profiles, albeit with similar brightness, for the different $\mathrm{x}$-ray production modes. The parameters for the injector are listed in Table 1.

\begin{tabular}{|lll|}
\hline Energy at rf gun exit & $\sim 10 \mathrm{MeV}$ \\
Energy at compressor entrance & $\sim 120 \mathrm{MeV}$ \\
Bunch charge & $1-3 \mathrm{nC}$ \\
Flat beam injector $(1 \mathrm{nC}):$ & & \\
$\quad$ Horizontal emittance & $\sim 20 \mathrm{~mm}-\mathrm{mrad}$ \\
$\quad$ Vertical emittance & $<0.4 \mathrm{~mm}-\mathrm{mrad}$ \\
$\quad$ Total emittance & $<3 \mathrm{~mm}-\mathrm{mrad}$ \\
Round beam injector $(1 \mathrm{nC}):$ & $<2 \mathrm{~mm}-\mathrm{mrad}$ \\
$\quad$ Emittance & $\pm 15 \mathrm{keV}$ \\
Uncorrelated energy spread & $\pm 600 \mathrm{keV}$ \\
Correlated energy chirp & $\sim 20 \mathrm{ps}$ \\
Bunch length at compressor entrance & $2 \mathrm{ps}$ \\
Bunch length at compressor exit & $\sim 10+\mathrm{kHz}$ \\
Repetition rate &
\end{tabular}

Table 1: LUX photoinjector parameters.

The dual-gun injector complex is shown schematically in Figure 1. Two rf photoinjector guns [3] provide time-

*This work was supported by the Department of Energy under Contract No. DE-AC03-76SF00098.

\# Electronic mail: SMLidia@lbl.gov interleaved beams at the $10 \mathrm{kHz}$ repetition rate. One gun utilizes a magnetized cathode configuration for flat beam production [4], while the other gun produces a typical 'round' beam from an unmagnetized cathode. The beams from the two guns are both injected into the superconducting linac and from there follow an identical beamline. The magnetized beam follows a linear trajectory to the linac, while the unmagnetized beam is transferred via an achromat transfer line from a gun situated off-axis.

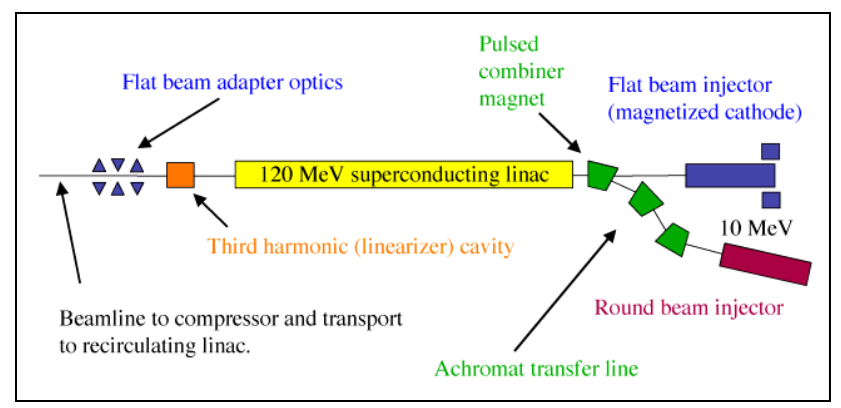

Figure 1: LUX dual-gun injector complex.

Following the linac, a $3^{\text {rd }}$ harmonic $(3.9 \mathrm{GHz})$ cavity $(3 \mathrm{HC})$ acts to linearize the longitudinal phase space of the beam, and to impose the requisite correlated energy chirp prior to injection to the compressor beamline and transfer to the main recirculating linac of LUX. Between the $3 \mathrm{HC}$ and the compressor is the 'Adapter' beamline of skew quadrupoles followed by normal quadrupoles. The skew quadrupole lattice removes the angular momentum carried by the magnetized ('flat') beam, producing a beam with large $(\sim 50)$ ratio between horizontal and vertical emittance. The normal quadrupoles then match the beam to the compressor.

\section{BEAMLINE OPTIMIZATION}

We have optimized the generation, transport, acceleration, and manipulation of the electron beams using the 2.5D particle tracking code Astra [5]. Optimization seeks to find beamline parameters that simultaneously produce both sets of beams ('flat', 'round') which satisfy the performance requirements (Table 1). Considerable effort has been spent on studying emittance compensation [6] for beams carrying significant canonical angular momentum. The evolution of the magnetized beam is shown in Figure 2, and that of the unmagnetized beam in Figure 3. Figure 2 shows the evolution of several various normalized RMS 'emittances' projected over the full 20ps bunch length: the total 4D emittance $\left(\varepsilon_{4 \mathrm{D}}\right)$; the usual radial emittance $\left(\varepsilon_{\mathrm{r}}\right)$; and the two uncoupled, normal mode 
emittances $\left(\varepsilon_{\text {drift }}, \varepsilon_{\text {cyclotron }}\right)$. For eventual application of the flat beam where an ultra-low vertical emittance is required, the cyclotron emittance is minimized. For quasilaminar beams, the circular (normal) mode emittances are related to the radial emittance and canonical angular momentum $\left(\mathrm{M}=\mathrm{xp}_{\mathrm{y}}-\mathrm{yp} \mathrm{p}_{\mathrm{x}}\right)$ via

$$
2 \varepsilon_{ \pm}= \pm\langle M\rangle+\sqrt{\left(2 \varepsilon_{r}\right)^{2}+\left\langle M^{2}\right\rangle} \text {. }
$$

The drift and cyclotron (i.e. circular modes) emittances correspond to the $(+)$ and (-) angular momentum modes in Equation (1).

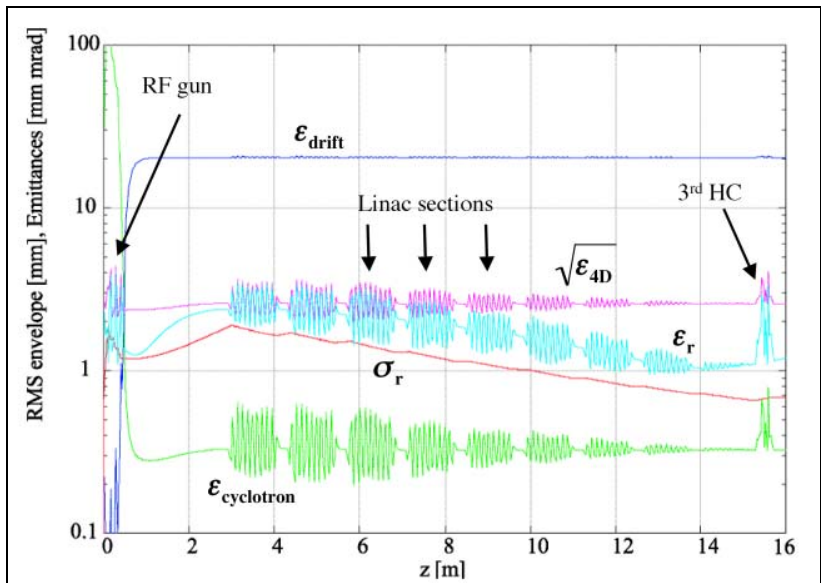

Figure 2: RMS spot and normalized emittance evolution from the cathode $(\mathrm{z}=0 \mathrm{~m})$ to the entrance of the Adapter $(\mathrm{z}=16 \mathrm{~m})$ for the magnetized ('flat') beam.

Until the symmetry of the beamline is broken in the skewquadrupole Adapter lattice, the canonical angular momentum of the beam is conserved. Hence, emittance compensation in this beamline can be monitored by observing the evolution of the radial emittance. Figure 2 illustrates that compensation of the cyclotron emittance through the linac tracks along with the radial emittance. The total 4D emittance (conserved for linear forces) remains essentially constant along this entire portion of the beamline, and at $\sim 2.5 \mathrm{~mm}$-mrad meets the injector specifications with some overhead.

The evolution of the unmagnetized beam up to the Adapter lattice is shown in Figure 3. The gun-to-linac drift length has been increased from $3 \mathrm{~m}$ to $5 \mathrm{~m}$ to match the phase of the emittance oscillation. In so doing, the radial emittance is compensated at the linac exit, also with some overhead compared to specifications.

\section{FLAT BEAM ADAPTER OPTICS}

Following the $3^{\text {rd }}$ harmonic cavity is the Adapter lattice consisting of 3 skew quadrupoles. The spacing and excitation strength of the skew quadrupoles are determined [7] to cancel the correlations induced by the canonical angular momentum and decouple the horizontal and vertical phase spaces. When this is done properly, the residual angular momentum is removed and, most importantly, the drift and cyclotron emittances are mapped to the horizontal and vertical, producing a flat beam. The evolution of the horizontal and vertical beam emittances and spot sizes are shown in Figure 4.

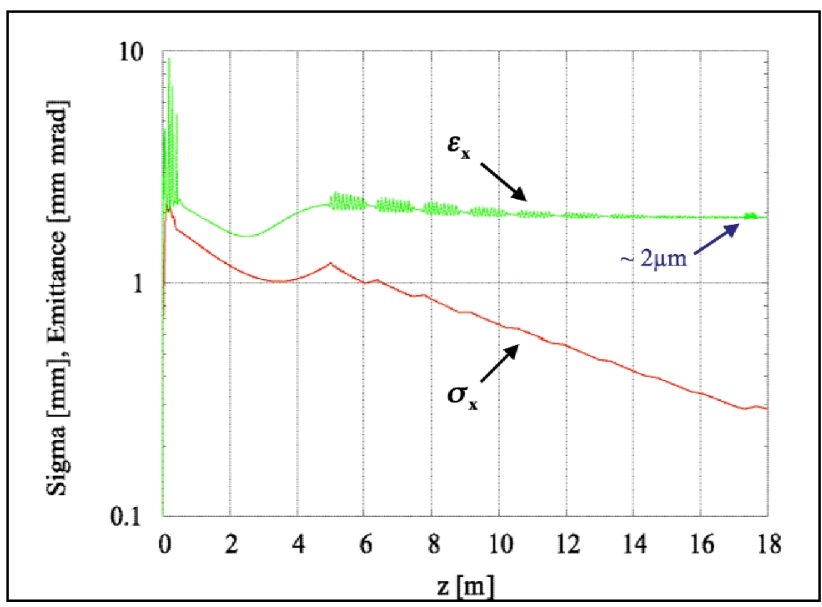

Figure 3: RMS spot and normalized emittance evolution from the cathode to the entrance of the Adapter for the unmagnetized ('round') beam.

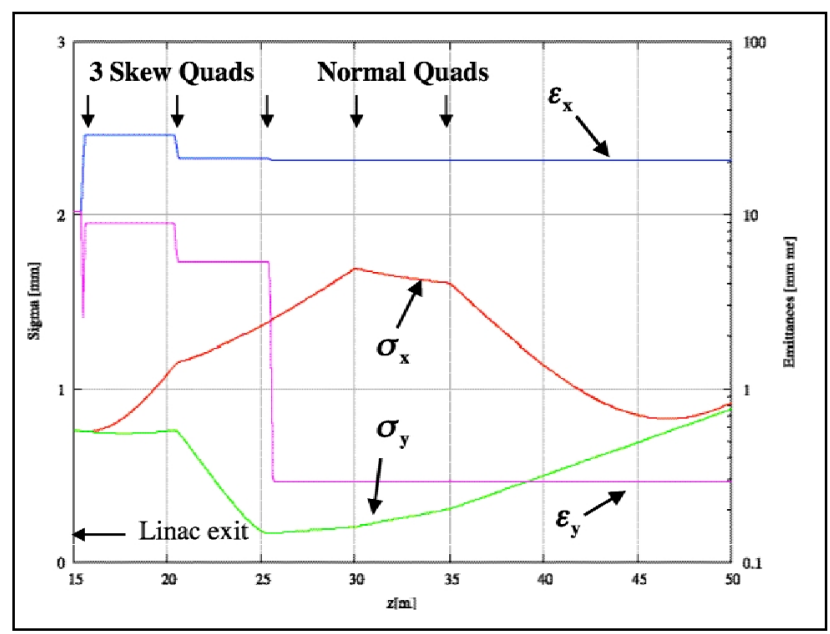

Figure 4: Horizontal and vertical RMS spot size and normalized emittance evolution in the Adapter.

\section{LONGITUDINAL DYNAMICS AND BUNCH COMPRESSION}

Manipulation of the longitudinal phase space is another essential duty of the injector. To offset intense spacecharge forces at low energy, the beam is generated at the cathode with a bunch length $\sim 20$ ps. The launch phase and cell-to-cell phasing in the rf guns are adjusted to produce a low emittance beam with small uncorrelated energy spread. The linac produces a nonlinear correlation over the bunch length, which is removed with the $3^{\text {rd }}$ harmonic cavity. The evolution of the longitudinal phase space from the $1^{\text {st }}$ rf gun cell to the gun exit to the linac exit and the $3^{\text {rd }}$ harmonic cavity exit is shown in Figure 5. As shown, the longitudinal phase space at the entrance to the compressor has an uncorrelated energy spread within specifications. 


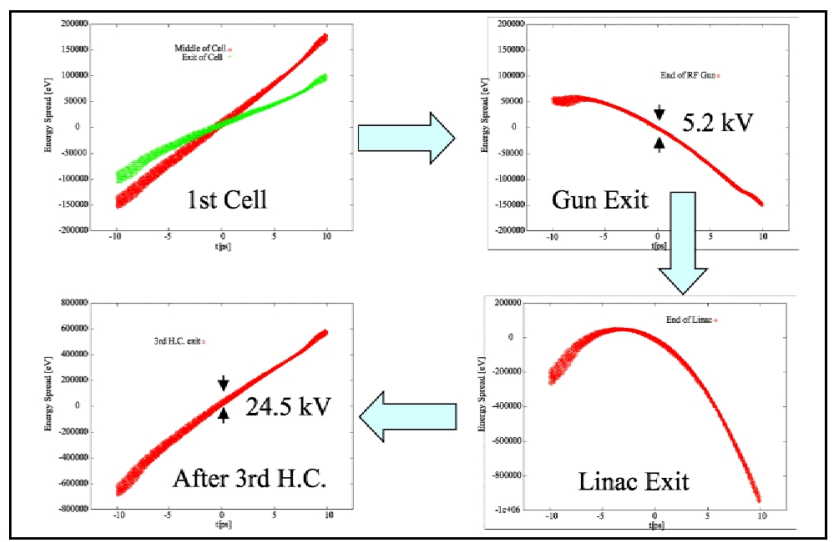

Figure 5: Development of longitudinal phase space from the rf gun to the compressor entrance.

After the flat beam adapter the electron beam executes a $180^{\circ}$ turn before it merges the recirculating linac in the bending magnet of the injection chicane (see Figure 6). We use this $180^{\circ}$ turn to compress the electron bunch from $\sim 20-30$ ps to approximately 2 ps. The design is largely affected by considerations regarding detrimental effects of CSR (coherent synchrotron radiation). Following [8] we split the actual bunch compressor into two parts and separate them by $-I$ transport lines. The idea is to use the CSR in the downstream bunch compressor to compensate for the CSR induced excitation of the horizontal emittance in the upstream bunch compressor. This will give us only a limited success because of the difference in bunch lengths in the upstream and downstream bunch compressors. Therefore to improve compensation we choose bending radii for bend magnets in the downstream compressor to be 1.5 times larger than in the upstream compressor.

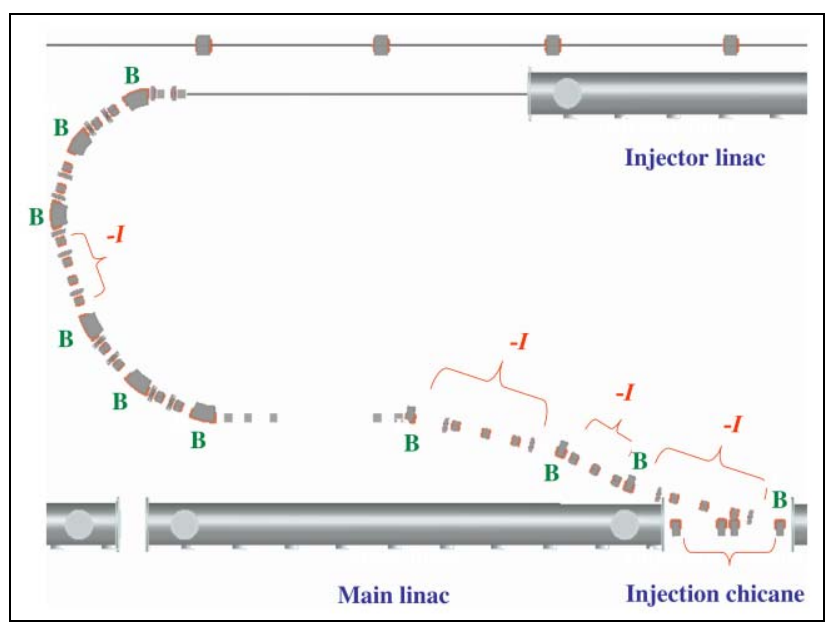

Figure 6: Compressor beamline.

The effect of CSR on horizontal emittance is strongest in the final bend. This is because of the large betafunction in the recirculating linac at the injection point. Compensation of CSR in this magnet requires more complicated arrangements for injection than just a bend. We found that we can have a fairly effective compensation using four bend magnets and separate them by $-I$ transport matrix. In this setting, the two upstream bends have the opposite polarity to the two downstream bends. An additional benefit is that the overall arrangement is achromatic.

To analyze this design we performed particle tracking through the entire bunch compressor and injection lattice using elegant [9]. An initial particle distribution was loaded that is uniform both transversely and longitudinally. Tracking through the compressor beamline shows a relative horizontal emittance gain less than $25 \%$, with no discernable increase in energy spread.

\section{CONCLUSIONS}

We have produced an injector design for LUX that exceeds performance specifications. There are still several areas that need additional study. We must predict any emittance growth in the low energy achromat line, as well as incorporate its optics into the general emittance compensation scheme for the unmagnetized beam. The Adapter beamline has been optimized for the production of low vertical emittance flat beams using a simple skew quadrupole triplet configuration. This beamline must perform this duty while also matching both the flat and round beam into the compressor. A more complicated beamline may be required to perform this job, and must be studied further.

\section{REFERENCES}

[1] J. Corlett, et. al., "A Recirculating Linac-Based Facility for Ultrafast X-ray Science", Proc. 2003 Part. Accel. Conf., Portland, 2003.

[2] W. Fawley, et. al., "Simulation Studies of an XUV/Soft X-Ray Harmonic-Cascade FEL for the Proposed LBNL Recirculating Linac", Proc. 2003 Part. Accel. Conf., Portland, 2003.

[3] J. Staples, et. al., "The LBNL Femtosource $10 \mathrm{kHz}$ Photoinjector", Proc. 2003 Part. Accel. Conf., Portland, 2003.

[4] R. Brinkmann, Y. Derbenev, and K. Floettmann, TESLA Note 99-09, April 1999. A.Burov, Y. Derbenev, S. Nagaitsev, Phys. Rev. E 66, 016503 (2002).

[5] Astra executables and documentation may be obtained at http://www.desy.de/ mpyflo.

[6] B.E. Carlsten, NIM A 285, 313 (1989). L. Serafini and J.B. Rosenzweig, Phys. Rev. E 55, 7565 (1997). S. Lidia, "Emittance Compensation Studies of Photoinjector Beams with Angular Momentum", Proc. 2003 Part. Accel. Conf., Portland, 2003.

[7] E. Thrane, et. al., "Photoinjector Production of a Flat Electron Beam", Proc. XXI Int'l. Linear Accel. Conf., Gyeongju, Korea, 2002.

[8] Linac Coherent Light Source, Conceptual Design Report, SLAC-R-593, UC-414, (2002).

[9] M. Borland, "elegant: A Flexible SDDS-Compliant Code for Accelerator Simulation", Proc. Int'l. Comp. Accel. Physics Conf., Darmstadt, Germany, 2000. 